\title{
Imaging three-dimensional polarization in epitaxial polydomain ferroelectric thin films
}

\author{
C. S. Ganpule, ${ }^{\text {a) }}$ V. Nagarajan, B. K. Hill, A. L. Roytburd, E. D. Williams, \\ and R. Ramesh ${ }^{\mathrm{b})}$ \\ Materials Science Research and Engineering Center, University of Maryland, College Park, Maryland 20742 \\ S. P. Alpayc) \\ Department of Metallurgy and Materials Engineering, University of Connecticut, Storrs, Connecticut 06269
}

\author{
A. Roelofs and R. Waser \\ Institut für Werkstoffe der Elektrotechnik, RWTH Aachen, D-52056 Aachen, Germany \\ L. M. Eng \\ Institut für Angewandte Photophysik, Technische Universität Dresden, D-01062 Dresden, Germany
}

(Received 13 June 2001; accepted for publication 1 October 2001)

\begin{abstract}
Voltage-modulated scanning force microscopy (Piezoresponse microscopy) is applied to investigate the domain structure in epitaxial $\mathrm{PbZr}_{0.2} \mathrm{Ti}_{0.8} \mathrm{O}_{3}$ ferroelectric thin films grown on (001) $\mathrm{SrTiO}_{3}$. $\mathrm{By}$ monitoring the vertical and lateral differential signals from the photodetector of the atomic force microscope it is possible to separate out and observe the out-of-plane and in-plane polarization vectors in the thin film individually. The relative orientation of the polarization vectors across a $90^{\circ}$ domain wall is observed. Nucleation of new reversed $180^{\circ}$ domains at the $90^{\circ}$ domain wall is studied and its impact on the rotation of polarization within the $a$ domain is analyzed as a function of reversal time. (C) 2002 American Institute of Physics. [DOI: 10.1063/1.1421219]
\end{abstract}

\section{INTRODUCTION}

Investigation of the domain structure and its evolution under an electrical field is important in understanding the macroscopic behavior of ferroelectric materials for applications in nonvolatile memories, sensors, and actuators. ${ }^{1}$ Several experimental techniques have been developed in the past to reveal this domain structure, such as optical microscopy, pyroelectric detection, surface etching, x-ray topography, colloidal decoration, and electron microscopy among others. ${ }^{2}$ These methods however suffer from some serious limitations, either in terms of sample preparation conditions (destructive) or in terms of the lateral resolution achievable. Advances in scanning probe techniques have, in recent years, led to a dramatic improvement in both the ease of sample preparation and the absolute resolution available in imaging the ferroelectric domain structure, thereby providing the tools necessary for nanoscale domain observation and manipulation. ${ }^{3}$ Most of these studies have focused on observation of the vertical vibration of the atomic force microscope (AFM) cantilever, thereby tracking the out-of-plane polarization vector. Eng et al., ${ }^{4,5}$ Abplanalp et al., ${ }^{6}$ and Eng et al., ${ }^{7}$ have recently shown that the in-plane component of the polarization can be observed by following the torsion or lateral deflection of the AFM cantilever and have applied this technique to reconstruct the three-dimensional distribution of polarization within domains of ferroelectric single crystals.

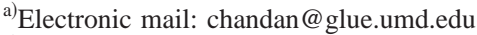

b) Author to whom correspondence should be addressed; electronic mail: rr136@umail.umd.edu

${ }^{c)}$ Electronic mail: p.alpay@ims.uconn.edu
Roelofs et al. applied this method in order to differentiate $90^{\circ}$ and $180^{\circ}$ domain switching in $\mathrm{PbTiO}_{3}$ thin films. ${ }^{8}$

In this article, we have applied voltage-modulated scanning force microscopy to the visualization of threedimensional domain structure in epitaxial $\mathrm{PbZr}_{0.2} \mathrm{Ti}_{0.8} \mathrm{O}_{3}$ (PZT) ferroelectric thin films grown on single crystal (001) $\mathrm{SrTiO}_{3}$ substrates. Tetragonal PZT thin films show the $c / a / c / a$ polydomain pattern that consists of alternating $c$ domains with the tetragonal axis perpendicular to the filmsubstrate interface, and $a$ domains (90 domains) with the $c$ axis of the tetragonal film along either [100] or [010] directions of the substrate. ${ }^{9}$ The formation of polydomain structures in ferroelectric films is a mechanism of strain energy relaxation. ${ }^{10}$ It is especially important to visualize and study such domain formation in ferroelectrics since it can have a profound influence on the physical properties of these materials and hence on the switching behavior and reliability issues like retention loss. In particular, it becomes important to study the orientation of the polarization vector in the $a$ domains of the film when the adjoining $c$ domains are switched. Merz had proposed the head-tail alignment of polarization vectors at the interface of $a$ and $c$ domains to prevent charge building up at the boundary and had made optical observations of the same. ${ }^{11,12}$ However, these studies were conducted on bulk $\mathrm{BaTiO}_{3}$ single crystals and may not necessarily represent the situation in epitaxial thin film samples. We have recently shown the role of $a$ domains in the nucleation of reverse $180^{\circ} c$ domains. ${ }^{9}$ It is necessary to study both the orientation of the polarization vectors across a $90^{\circ}$ wall in the as grown film and the effect of $180^{\circ}$ polarization rotation of the $c$ domains on the rotation of polarization in the $a$ domains. 


\section{EXPERIMENT}

The ferroelectric films consisted of epitaxial $400 \mathrm{~nm}$ thick PZT films grown on a single crystal $\mathrm{SrTiO}_{3}$ (STO) substrate with a $50 \mathrm{~nm}$ thick $\mathrm{La}_{0.5} \mathrm{Sr}_{0.5} \mathrm{CoO}_{3}$ (LSCO) oxide layer as the bottom electrode. PZT thin films were deposited at $650{ }^{\circ} \mathrm{C}$ and 100 mTorr oxygen partial pressure on (001) epitaxial grade STO substrates (Crystec GmbH, Germany) by pulsed laser deposition. The samples were cooled at a rate of $1{ }^{\circ} \mathrm{C} / \mathrm{min}$ from the growth temperature. The thicknesses of films were confirmed by Rutherford backscattering and transmission electron microscopy (TEM). Epitaxial growth in the samples was established from $\phi$ scans and the presence of only $00 l$ type reflections in the $\theta-2 \theta$ x-ray diffraction (XRD) pattern.

The SFM piezoresponse mode has been effectively applied for visualization of domain structure in PZT thin films. ${ }^{3-9}$ It is based on the detection of the local electromechanical vibration of the ferroelectric sample caused by an external ac voltage. The voltage is applied through the probing tip, which is used as a movable top electrode. The modulated deflection signal from the can tilever, which oscillates together with the sample, is detected using the lock-in technique. In the piezoresponse mode the frequency of the imaging voltage should be far lower than the cantilever resonant frequency to avoid mechanical resonance of the cantilever. An external voltage with a frequency $\omega$ causes a sample vibration with the same frequency due to the converse piezoelectric effect. Vibration of the sample under the ac voltage also has a second harmonic component at $2 \omega$ due to the electrostrictive effect and dielectric constant. The domain structure can be visualized by monitoring the first harmonic signal (piezoresponse signal). The phase of the piezoresponse signal depends on the sign of the piezoelectric coefficient (and therefore on the polarization direction) and reverses when the coefficient is opposite. This means that regions with opposite orientation of polarization, vibrating in counter phase with respect to each other under the applied ac field, should appear as regions of opposite contrast in the piezoresponse image.

For our experiments, we used a commercial Digital Instruments Nanoscope IIIA Multimode scanning probe microscope equipped with standard silicon tips coated with $\mathrm{Pt} / \mathrm{Ir}$ alloy for electrical conduction. The typical force constant of these tips was $5 \mathrm{~N} / \mathrm{m}$ and the apex radius was approximately $20 \mathrm{~nm}$. The contact force was estimated to be around $70-100 \mathrm{nN}$.

\section{RESULTS AND DISCUSSION}

The domains in epitaxial ferroelectric films are tilted by a small angle with respect to the film-substrate normal. ${ }^{13-15}$ The theoretical tilt angle can easily be determined from the geometry of the polydomain structure [see Fig. 1(a)] as

$$
\omega=2 \tan ^{-1}\left(\frac{c}{a}\right)-\frac{\pi}{2}
$$

The tetragonality of bulk PZT is 1.05 at room temperature, corresponding to a theoretical tilt angle of $\sim 2.9^{\circ}$. Experimental results show that this theoretically predicted tilt of $a$
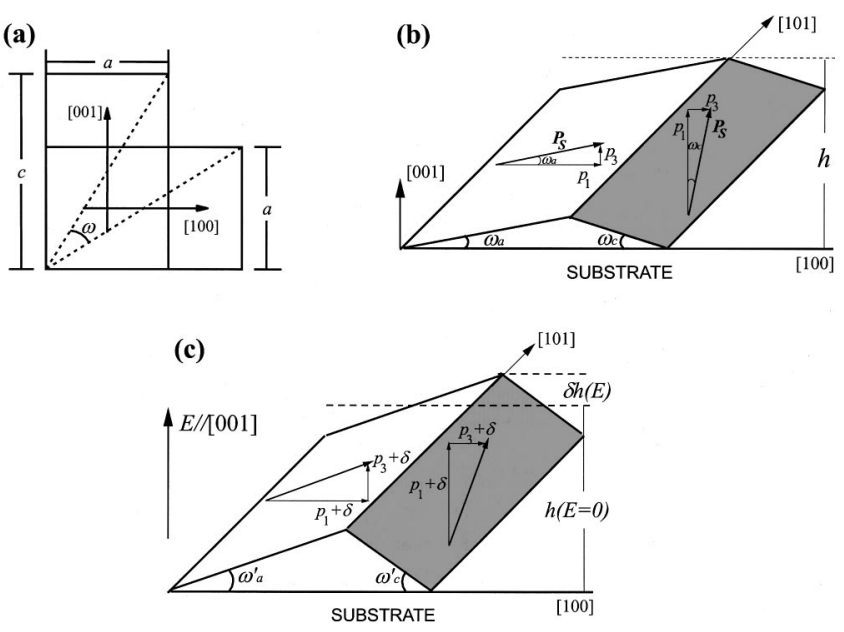

FIG. 1. (a) The tilt in polydomain films consisting of $a$ and $c$ domains when brought together at the (101) interface due to the tetragonality of the lattice. (b) The accommodation of tilt in polydomain films consisting of $a$ and $c$ domains. (c) The change in the tilt angles due to applied field $E \|[001]$. Tilt angles are highly exaggerated. $\delta$ in both domains should be the same to maintain mechanical and electrical compatibility across the domain wall.

domains is not observed ${ }^{14,15}$ and is always less than $\omega$. The reason for this is that the tilt is accommodated in both $a$ and $c$ domains, depending on their volume fractions. The tilt accommodation is schematically illustrated in Fig. 1(b). From the geometry, the tilts in each domain are

$$
\frac{\tan \omega_{a}}{\tan \omega_{c}}=\frac{\alpha}{1-\alpha},
$$

where $\alpha$ is the fraction of $c$ domains, and $\omega_{a}$ and $\omega_{c}$ are the tilts in the $a$ domains and $c$ domains, respectively. ${ }^{16}$ This domain structure results in a fourfold tilt [rather than the simple tilt as shown in Fig. 1(b) of the (h00) and (00l) planes away from the $[00 l]$ direction of the substrate along [h00], [ $\bar{h} 00],[0 k 0]$, and [0 $\bar{k} 0]$.] The fourfold splitting of the $a$ and $c$ domains is readily observed via x-ray diffraction in $\theta$ rocking curves ${ }^{13,16,17}$ and $\theta-\chi$ scans (area maps). ${ }^{14,15,18,19}$ The fourfold symmetry of $c$ domains is less pronounced, but nonetheless existent, for films with a high fraction of $c$ domains since the angle $\omega_{c}$ is small. ${ }^{14,19}$

In a previous study, ${ }^{20}$ a sample identical to the one used in this work was analyzed crystallographically by XRD. The $c$-domain fraction was determined to be 0.80 and the $a$ domains were found to be tilted from the [001] peak of the substrate by $2.3^{\circ}$. This means that the $a$ domains possess a small component of polarization $p_{3}$ along the [001] direction as shown in Fig. 1(b). If an electrical field is applied parallel to the [001] direction, $p_{3}$ increases by $\delta$, which causes a rotation of the polarization vector in the (010) plane [Fig. $1(c)]$. This in turn results in elastic shear deformation characterized by the piezocoefficient $d_{15}$. Taking into account this shear deformation, the tip will correspondingly be pushed by it or dragged with it, giving rise to the asymmetric behavior displayed in Figs. 1(b) and 1(c). It should be noted that to maintain mechanical and electrical compatibility across the domain wall, $\delta$ should be identical in both $a$ and $c$ domains. 

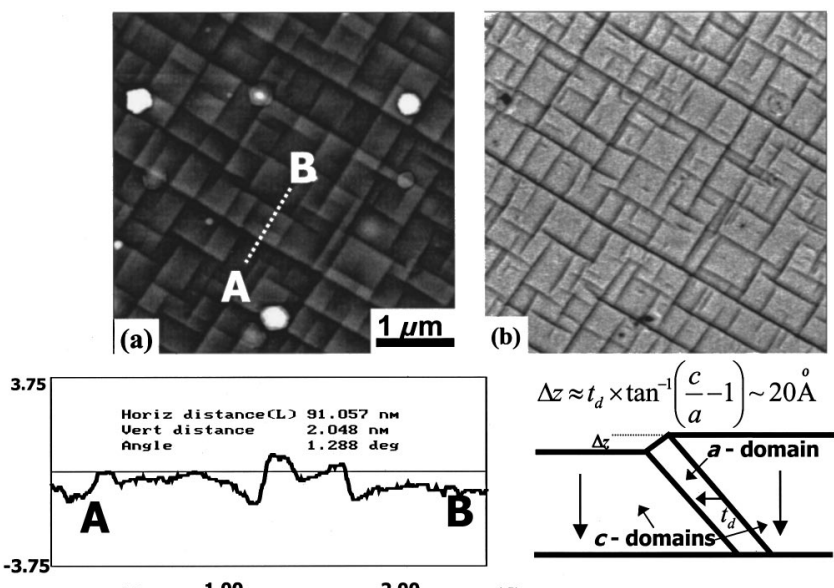

(c) $\mu \mathrm{m}$

(d)

FIG. 2. (a) Topography and (b) piezoresponse (vertical differential signal) images taken simultaneously across the PZT thin film. A line scan across $A B$ shows the surface relief across $a$ domains. A schematic of the relief across an $a$ domain is shown in (d).

Figure 2(a) shows the surface relief of the PZT film. Figure 2(b) plots a topographic line scan across a pair of $90^{\circ}$ domains. The vertical relief across a single $a$ domain is approximately $2 \mathrm{~nm}$. A schematic of the surface relief as seen in the line scan in Fig. 2(b) is sketched in Fig. 2(c). The domain image of the same region [from (a)] as seen in the piezoresponse Fig. 2(d) shows that the $c$-axis oriented regions of the film are highly oriented. No top electrodes were deposited in the films investigated. By leaving the top ferroelectric layer exposed to allow the tip direct access (in order to optimize our signal-to-noise ratio), we effectively use the tip as the top electrode of the capacitor. This built-in asymmetry leads to a preferred energy state for one polarization orientation over the other ${ }^{9}$ as can be seen in Fig. 2(d). The piezoresponse image in Fig. 2(d) was acquired by tracking the differential vertical displacement of the cantilever. In this mode of imaging, the interlocking grid of orthogonal $a$ domains can be seen as regions of low piezoresponse. It is possible to observe from Fig. 2(d) that while some $a$ domains are long and wide, others appear short and thin. The TEM cross sectional image (Fig. 3) of a similar sample shows evidence for primary twins, i.e., twins that nucleate at the bottom electrode-ferroelectric interface and propagate to the top surface, and secondary twins that nucleate on primary twins and grow to the surface.

In order to map the polarization vectors in these $a$ domains, the lateral differential signal from the photodiode was tracked. This signal effectively represents torsion deformation of the AFM cantilever. As the tip scans over the $a$-domain regions, the electrical field through the tip produces the shearing deformation mentioned earlier, giving rise to the torsion signal in the photodiode. This is schematically illustrated in Figs. 4(a) and 4(b) for oppositely oriented $a$ domains. The phase of this torsion motion of the tip with respect to the input sinusoidal electric field provides information about the orientation of polarization in the domain. It should be pointed out that the scan direction of the tip is chosen to be such that the orthogonal $a$ domains contribute

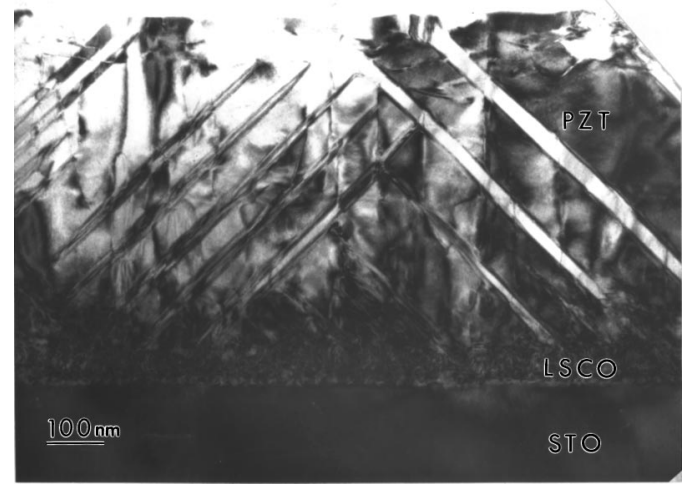

FIG. 3. Cross-sectional TEM micrograph of a $400 \mathrm{~nm}$ thick PZT film grown on a single crystal $\mathrm{SrTiO}_{3}$ (STO) substrate with a $50 \mathrm{~nm}$ thick LSCO oxide layer as the bottom electrode. Primary twins are seen to nucleate at the bottom electrode-ferroelectric interface while secondary twins are seen to nucleate along primary twins.

equally to the tip motion. The tip, therefore, scans at $45^{\circ}$ to the $a_{1}$ and $a_{2}$ domains.

Figures 5(a) and 5(b) show typical out-of-plane and inplane piezoresponse images of the same region in the PZT film. In an earlier publication we showed that it was possible to find out the inclination of the $a$ domain with respect to the substrate by taking a line scan of the piezoresponse signal across the domain. ${ }^{9}$ By combining this information with the knowledge about polarization direction within the $a$ domain as seen in Fig. 5(b), it is possible to see that the head to tail configuration of the polarization vectors is maintained across the $90^{\circ}$ domain wall. Thus domains aligned at $45^{\circ}$ and $135^{\circ}$ to the substrate are phase shifted by $180^{\circ}$ and therefore lead to different contrast levels in the in-plane image. Figure 5(c) plots a line scan of the vertical differential signal across $A B$ in Fig. 5(a), thereby implying that the domain is oriented as illustrated schematically in 5(c). Figure 5(d) plots a similar line scan across $C D$ in Fig. 5(a), thus showing that this $a$ domain is oppositely oriented with respect to the substrate. As expected the image contrast within these domains is reversed as noticed in the in-plane image (b).

By suitably biasing the scanning AFM tip it is possible to reverse the domain state within film. In Fig. 6(a) (out-ofplane piezoresponse image), the regions of dark contrast were poled into the opposite polarity by scanning the tip with a dc bias of $-8 \mathrm{~V}$. Figure 6(b) shows a corresponding

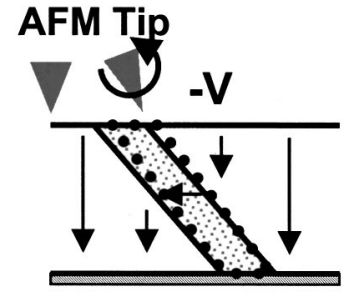

(a)

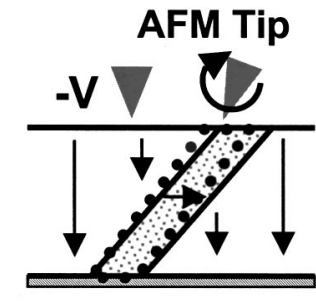

(b)
FIG. 4. Shearing deformation within $a$ domains oppositely oriented [(a) and (b)] giving rise to a torque motion of the AFM cantilever. 

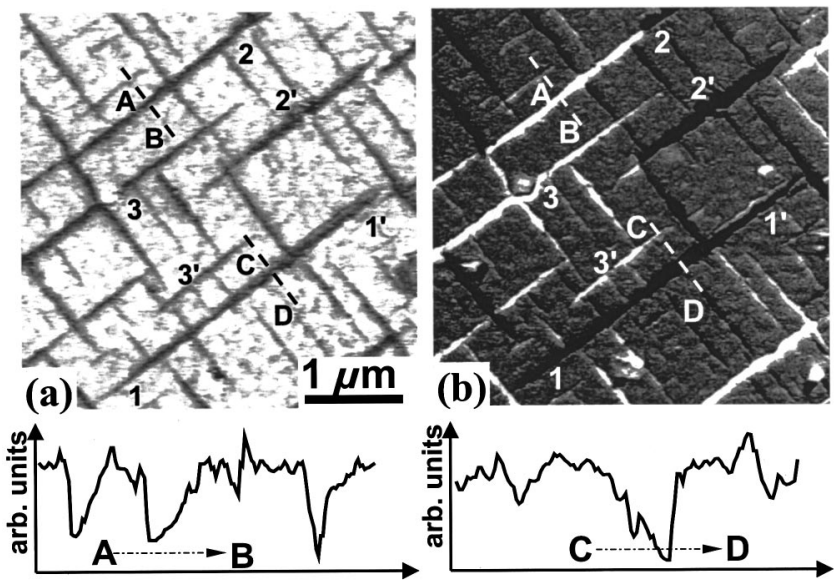

(c)

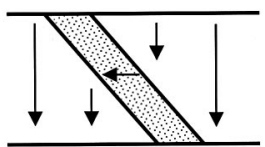

(d)

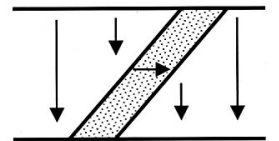

FIG. 5. (a) Out-of-plane (vertical differential signal) and (b) in-plane (lateral differential signal) images of PZT(0/20/80). (c) and (d) are line scans across regions marked $A B$ and $C D$ from (a) with schematic showing full reconstruction [with in-plane vector information from (b)] of domain structure and polarization orientation across oppositely oriented $a$ domains.

in-plane piezoresponse image. Thus, we effectively have created $180^{\circ}$ domain walls. If the tip is directly on top of a $180^{\circ}$ domain wall and the AFM cantilever aligned along the wall, the electric field penetrates regions of oppositely poled domains on either side of the cantilever. The differential piezoelectric activity in these domains causes the AFM cantilever

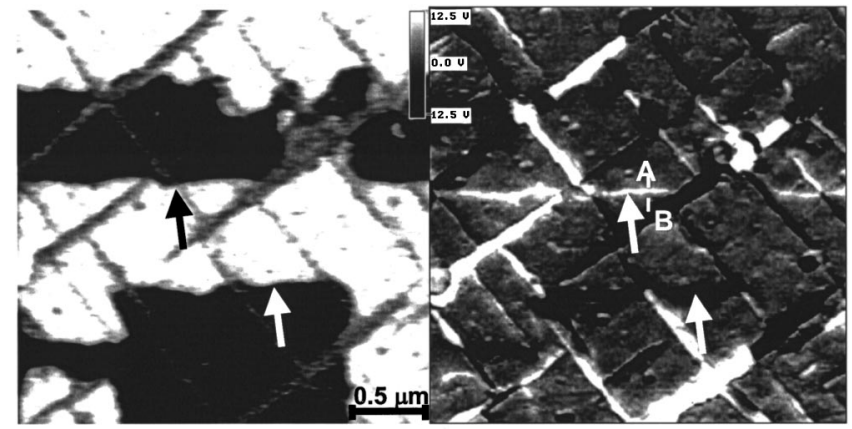

(a)

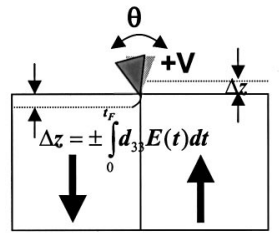

(b)

(c)

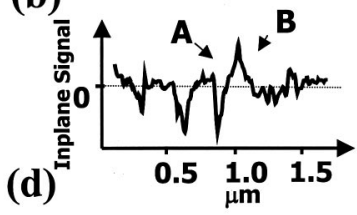

FIG. 6. (a) Out-of-plane (vertical differential signal) and (b) in-plane (lateral differential signal) images of PZT(0/20/80). Regions with dark contrast in (a) have been poled into the opposite direction by scanning the surface of the film selectively with $-8 \mathrm{~V}$. (c) shows a schematic of the tip deflection on top of a $180^{\circ}$ domain wall with the AFM cantilever aligned along the wall. Differential vertical displacements on either side of the wall give rise to a torque on the cantilever that can be seen by tracking the lateral differential signal from the photodetector. The presence of $180^{\circ}$ domain walls can therefore be seen in (b) as thin lines. (d) shows a line scan across one such domain wall marked $A B$ in (b).

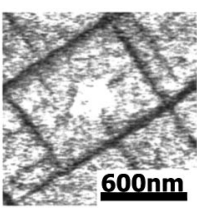

(a)

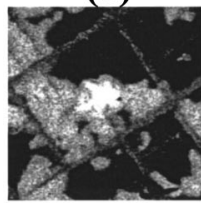

(e)

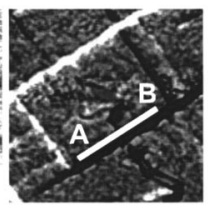

(b)

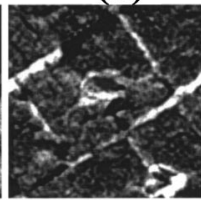

(f)

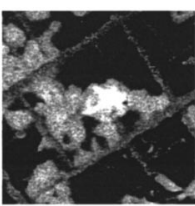

(c)

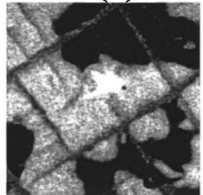

(g)

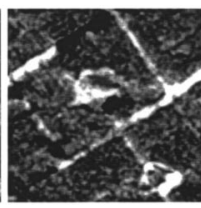

(d)

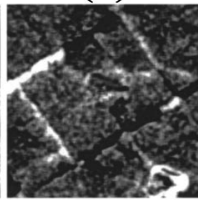

(h)
FIG. 7. Out of plane (a) and in-plane (b) domain images of PZT(0/20/80) in the as-grown condition. The same region was subsequently switched into the opposite polarization as seen in (c) (out-of-plane) and (d) (in-plane) images (taken after $t=1.8 \times 10^{4} \mathrm{~s}$ ). Out-of-plane images (e) and (g) and in-plane images (f) and (h) were taken after $t=2.6 \times 10^{4} \mathrm{~s}$ and $t=5.9 \times 10^{4} \mathrm{~s}$, respectively.

to torque as illustrated in Fig. 6(c). Therefore, it is in principle possible to image a single $180^{\circ}$ domain wall. However, the nonuniform field distribution below the tip and the geometry of the tip leads us to assume that the width of the $180^{\circ}$ domain wall $(20 \mathrm{~nm})$ as seen in the line scan of Fig. $6(d)$ is clearly an upper bound.

The absence of a top electrode in our samples leads to a favorable state of polarization along the $c$ axis as seen in Fig. 2(d) and 5(a). This stable state of polarization can be switched into the opposite unstable state as in Fig. 6(a). This unstable state decays over time and through nucleation and growth of the original stable state converts the switched region back into the as grown state of polarization. ${ }^{21}$ The nucleation of reversed domains has been seen to occur primarily along the $90^{\circ}$ domains. It is important to study whether this nucleation of reversed domains is accompanied by reversal within the $a$ domain. Figures 7(a) and 7(b) are in-plane and out-of-plane domain images of the PZT film before writing (as grown). The out-of-plane polarization state

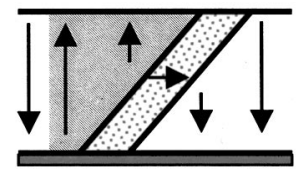

(a)

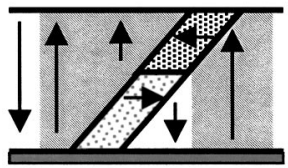

(c)

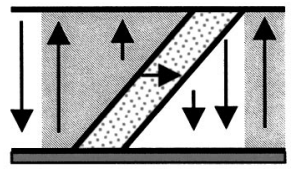

(b)

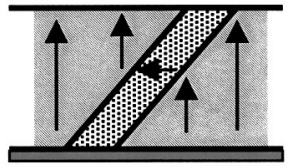

(d)
FIG. 8. Schematic illustrating that the in-plane polarization in the $a$ domain in (a) does not reverse with reverse $180^{\circ}$ domains on only one side of it (left). When the reverse $180^{\circ}$ domain on its right side (b) touches the $a$ domain, and in (c) moves closer toward it, the polarization within the $a$ domain reverses due to the electrostatic pressure on it from both sides as seen in (c) and (d). 
within the region scanned was switched into the opposite state by scanning with the tip biased at $-8 \mathrm{~V}$. The in-plane polarization within the $a$ domains was seen to switch at the same time, such that no charged interfaces could be identified. Images (c) and (d), (e) and (f), and (g) and (h) were acquired after wait times of $1.8 \times 10^{4}, 2.6 \times 10^{4}$, and 5.9 $\times 10^{4} \mathrm{~s}$, respectively. The reversed $c$ domains in (c) are seen to reach the $c / a$ domain interface $A B$ but no reversal of the in-plane polarization within the $a$ domain is observed. This interface therefore is likely to be charged as it represents a head-head arrangement of dipoles. The polarization within the $a$ domain is seen to reverse only after stable $c$ domains have nucleated on the opposite side of $A B$ as seen in (e) and grow to meet the interface $A B$ [in $(\mathrm{g})]$, thereby causing electrostatic pressure to be applied on both sides of the $a$ domain. This sequence, described above is illustrated in Figs. 8(a)$8(\mathrm{~d})$. It is possible that the sluggish nature of in-plane reversal under zero external field (relaxation) could be attributed to the small dimension (width) of the $a$ domain.

\section{SUMMARY}

To summarize, we have observed the complete threedimensional polarization within the PZT film. By tracking the lateral differential signal along with the vertical one, it becomes possible to image the polarization vectors across the $90^{\circ}$ domain wall. A single $180^{\circ}$ domain wall has been imaged by scanning the tip along the wall and tracking the torque on the cantilever produced by the differential vertical displacements on the two domains across the domain wall. Observations on the movement of a single $180^{\circ}$ domain wall across an $a$ domain during polarization relaxation indicate that there is correlated polarization reversal across the $c / a$ domain interface.

\section{ACKNOWLEDGMENTS}

The authors thank H. Li for the TEM image and gratefully acknowledge the support by NSF-MRSEC under Grant
No. DMR-0080008 and by the NSF under Grant No. DMR9903279.

${ }^{1}$ D. Damjanovic, Rep. Prog. Phys. 61, 1267 (1998).

${ }^{2}$ M. E. Lines and A. M. Glass, Principles and Applications of Ferroelectric and Related Materials (Clarendon, Oxford, 1977).

${ }^{3}$ P. Güthner and K. Dransfeld, Appl. Phys. Lett. 61, 1137 (1992); A. Gruverman, O. Auciello, and H. Tokumoto, ibid. 69, 3191 (1996); C. H. Ahn, T. Tybell, L. Antognazza, K. Char, R. H. Hammond, M. R. Beasely, $\emptyset$ Fischer, and J.-M. Triscone, Science 276, 1100 (1997); T. Tybell, C. H. Ahn, and J.-M. Triscone, Appl. Phys. Lett. 72, 1454 (1998); O. Auciello, A. Gruverman, H. Tokumoto, S. A. Prakash, S. Aggarwal, and R. Ramesh, Mater. Res. Bull. 23, 33 (1998).

${ }^{4}$ L. M. Eng, H.-J. Güntherodt, G. Roseman, A. Skliar, O. Oron, M. Katz, and D. Eger, J. Appl. Phys. 83, 5973 (1998).

${ }^{5}$ L. M. Eng, H.-J. Güntherodt, G. A. Schneider, U. Köpke, and J. Muñoz Saldaña, Appl. Phys. Lett. 74, 233 (1999).

${ }^{6}$ M. Abplanalp, L. M. Eng, and P. Günter, Appl. Phys. A: Mater. Sci. Process. 66A, S231 (1998).

${ }^{7}$ L. M. Eng, M. Abplanalp, and P. Günter, Appl. Phys. A: Mater. Sci. Process. 66A, S679 (1998).

${ }^{8}$ A. Roelofs, U. Böttger, R. Waser, F. Schlaphof, S. Trogisch, and L. M. Eng, Appl. Phys. Lett. 77, 3444 (2000).

${ }^{9}$ C. S. Ganpule, V. Nagarajan, H. Li, A. S. Ogale, D. E. Steinhauer, S. Aggarwal, E. D. Williams, R. Ramesh, and P. De Wolf, Appl. Phys. Lett. 77, 292 (2000).

${ }^{10}$ S. P. Alpay and A. L. Roytburd, J. Appl. Phys. 83, 4714 (1998); A. L. Roytburd, S. P. Alpay, L. A. Bendersky, V. Nagarajan, and R. Ramesh, ibid. 89, 553 (2001).

${ }^{11}$ W. J. Merz, Phys. Rev. 95, 690 (1954).

${ }^{12}$ E. Fatuzzo and W. J. Merz, Ferroelectricity (North-Holland, Amsterdam, 1967).

${ }^{13}$ B. S. Kwak, A. Erbil, J. D. Budai, M. F. Chrisholm, L. A. Boatner, and B. J. Wilkens, Phys. Rev. B 49, 14865 (1994).

${ }^{14}$ C. M. Foster et al., J. Appl. Phys. 78, 2607 (1995).

${ }^{15}$ C. M. Foster, W. Pompe, A. C. Daykin, and J. S. Speck, J. Appl. Phys. 79, 1405 (1996).

${ }^{16}$ Y. M. Kang, J. K. Ku, and S. Baik, J. Appl. Phys. 78, 2601 (1995).

${ }^{17}$ Y. M. Kang and S. Baik, J. Appl. Phys. 82, 2532 (1997).

${ }^{18}$ W.-Y. Hsu and R. Raj, Appl. Phys. Lett. 67, 792 (1995).

${ }^{19}$ B. M. Yen and H. Chen, J. Appl. Phys. 85, 853 (1998).

${ }^{20}$ S. P. Alpay, V. Nagarajan, L. A. Bendersky, M. D. Vaudin, S. Aggarwal, R. Ramesh, and A. L. Roytburd, J. Appl. Phys. 85, 3271 (1999).

${ }^{21}$ C. S. Ganpule, V. Nagarajan, S. B. Ogale, A. L. Roytburd, E. D. Williams, and R. Ramesh, Appl. Phys. Lett. 77, 3275 (2000). 